\title{
CLASSICAL YANG-BAXTER EQUATION AND LEFT INVARIANT AFFINE GEOMETRY ON LIE GROUPS
}

\author{
ANDRE DIATTA円 AND ALBERTO MEDINA
}

\begin{abstract}
Let $G$ be a Lie group with Lie algebra $\mathcal{G}:=T_{\epsilon} G$ and $T^{*} G=\mathcal{G}^{*} \rtimes G$ its cotangent bundle considered as a Lie group, where $G$ acts on $\mathcal{G}^{*}$ via the coadjoint action. We show that there is a 1-1 correspondance between the skew-symmetric solutions $r \in \wedge^{2} \mathcal{G}$ of the Classical Yang-Baxter Equation in $G$, and the set of connected Lie subgroups of $T^{*} G$ which carry a left invariant affine structure and whose Lie algebras are lagrangian graphs in $\mathcal{G} \oplus \mathcal{G}^{*}$.

An invertible solution $r$ endows $G$ with a left invariant symplectic structure and hence a left invariant affine structure. In this case we prove that the Poisson Lie tensor $\pi:=r^{+}-r^{-}$is polynomial of degree at most 2 and the double Lie groups of $(G, \pi)$ also carry a canonical left invariant affine structure.

In the general case of (non necessarly invertible) solutions $r$, we supply a necessary and suffisant condition to the geodesic completness of the associated affine structure 2 .
\end{abstract}

\section{Introduction-Summary}

Let $G$ be a Lie group whose Lie algebra is denoted by $\mathcal{G}:=T_{\epsilon} G$, where $\epsilon$ stands for the unit of $G$.

Since the end of 60s-the early 70s (see e.g. [4] ), it's well known that if $w^{+}$is a left invariant symplectic form on $G$, the formula

$$
\omega^{+}\left(\nabla_{x^{+}} y^{+}, z^{+}\right):=-\omega^{+}\left(y^{+},\left[x^{+}, z^{+}\right]\right)
$$

defines a (locally) flat and torsion free connection $\nabla$ (i.e an affine structure) in $G$ which is left invariant. Here $x^{+}$is the left invariant vector field satisfying $x_{\epsilon}^{+}=x$. Such a formula plays a crucial role in the study of symplectic and kählerian Lie groups developed in [17], [18], [19], [22], [6], [7].

The problem of finding those Lie groups (necessary solvable) which admit a geodesically complete, left invariant flat torsion free connection, is an open problem (see Milnor [23]), and few such Lie groups are known.

Left invariant affine structures on $G$ bijectively correspond to left symmetric algebra (LSA, also called Koszul-Vinberg algebra) structures on the Lie algebra $\mathcal{G}$ of $G$, compatible with bracket of $\mathcal{G}$ ( J.L. Koszul [14], [15]). Such structures first arised in the works of J.L. Koszul [14], [15], E.B. Vinberg [26], Y. Matsushima. Left symmetric algebras has been studied in Helmstetter, Medina, Dardié, Chu in [13], [21], [6], [7], [4] ... etc.

Later, M. Bordemann ( $[5]$ ) shows that a solution of the Classical Yang Baxter Equation (CYBE) $r \in \wedge^{2} \mathcal{G}$ always determines a left invariant affine structure on any Lie group $G^{*}$ whose Lie algebra is the dual $\mathcal{G}^{*}$ (relative to $r$ ) of $\mathcal{G}$. The corresponding left invariant connection on $G^{*}$, is given by the formula

$$
\nabla_{\alpha^{+}} \beta^{+}:=\left(a d_{r(\alpha)}^{*} \beta\right)^{+} .
$$

Let's identify the cotangent bundle $T^{*} G$ of $G$ and the trivial bundle $\mathcal{G}^{*} \times G$, by means of left translations. And let's endow this latter with a structure of semi-direct product group $\mathcal{G}^{*} \rtimes G$ of $G$ and the abelian Lie group $\mathcal{G}^{*}$, where $G$ acts in $\mathcal{G}^{*}$ by the coadjoint action.

Denote $\pi:=r^{+}-r^{-}$the Poisson Lie tensor associated to $r$, and $\mathcal{D}(\mathcal{G})$ the double Lie algebra of $(G, \pi)$. Here are some of the results we prove in this work:

- there is a bijective correspondance between the skew-symmetric solutions of the Classical Yang Baxter Equation $(\mathrm{CYBE})$ on $\mathcal{G}:=\operatorname{Lie}(G)$ and the connected Lie subgroups of $T^{*} G$ equipped with left invariant

1 The first author was partially supported by Enterprise Ireland.

2 Mathematics Subject Classification (2000): 53D17, 53A15, 17B62.

Key words and phrases: Yang-Baxter equation, Geodesically complete affine structures, Poisson geometry. 
affine structures whose Lie algebras are lagrangian graphs of a $\mathcal{G}$-valued skew-symmetric linear mapping of $\mathcal{G}^{*}$. Here the Lie algebra $t^{*} \mathcal{G}:=\mathcal{G}^{*} \rtimes \mathcal{G}$ of $T^{*} G$, is endowed with its canonical hyperbolic orthogonal structure ie its canonical dual pairing (theorem 2.0.1 ).

- let $r \in \wedge^{2} \mathcal{G}$ be a solution of the CYBE, then the affine structure defined by (2) in any dual Lie group $G^{*}$, is geodesically complete if and only if the symplectic leaf through $\epsilon$, of the left invariant Poisson structure $r^{+}$, is a unimodular (symplectic) Lie group (theorem 2.0.2). Thus, the geodesic completness of $G^{*}$ implies the solvability of $G^{*}$, thanks to a result due to Lichnerowicz-Medina [17].

In the case where $r$ is an invertible solution of the CYBE, we prove:

- the existence of left invariant affine structure, defined by $r$, on every connected Lie group $D(G)$ whose Lie algebra is the double Lie algebra $\mathcal{D}(\mathcal{G})$ (theorem 3.0.3). Such a structure is geodesically complete if and only if $G$ is unimodular (and solvable) ( see theorem 3.0.4).;

- the existence on $D(G)$ of a left invariant complex structure given by $r$ (proposition 3.0.1);

- that the Poisson Lie tensor $\pi=r^{+}-r^{-}$is polynomial of degree 2 (theorem 4.0.5).

Moreover, if the differential 2 -form $\omega^{+}$is exact, we prove that the symplectic leaves of $\pi$ are coverings over coadjoint orbits of $G$ (Corollary 4.2.1 ).

We would like to thank prof. D. V. Alekseevsky for various hepful discussions and comments.

\section{Reminders and notations.}

An affine structure on a manifold $M$, is given by a maximal atlas of charts (affine atlas) to an affine space with transition functions extending to affine transformations. Equivalently, an affine stucture on $M$ is given by an immersion, a so called developing map, from the universal cover $\tilde{M}$ of $M$ to an affine space (e.g $\mathbb{R}^{n}$ ), equivariant with respect to the so called holonomy representation $h: \pi_{1}(M) \rightarrow \operatorname{Aff}\left(\mathbb{R}^{n}\right)$, where $\pi_{1}(M)$ is the group of deck transformations of $\tilde{M}$.

A morphism of affine manifolds is a map whose expression in affine atlas extends to an affine map. An affine stucture in a Lie group $G$ is said to be left invariant if left translations are morphisms of the affine manifold $G$.

A Poisson manifold $M$ is a manifold whose ring of functions $\mathcal{C}^{\infty}(M)$ carries a Lie algebra bracket $\{$, which is a first order linear differential operator, in each of its arguments. The Hamiltonian vector field associated to an $f \in \mathcal{C}^{\infty}(M)$ is $X_{f}:=\{f,\}=.: \Lambda(d f,)=.: \Lambda^{\sharp}(d f)$, where $\Lambda$ is the associated bivector field. The tensor $\Lambda$ must satisfy $[\Lambda, \Lambda]=0$, here [,] is the so-called Schouten bracket ( see e.g [16]).

Every Poisson manifold foliates into symplectic manifolds (its symplectic leaves), which are the maximal integrals of the involutive distribution spanned by all the Hamiltonian vector fields. In general, it's a difficult problem to describe symplectic leaves of a Poisson manifold. One of the most important families of Poisson manifolds is the one of Poisson Lie groups.

A Poisson Lie group $(G, \pi)$ is a Lie group $G$ together with a Poisson structure $\pi$ such that the multiplication is a Poisson morphism. Set $[\alpha, \beta]_{*}:=d_{\epsilon}\{f, g\}$, where $\alpha:=d_{\epsilon} f$ and $\beta:=d_{\epsilon} g \in \mathcal{G}^{*}$ are the respective differentials of $f, g \in \mathcal{C}^{\infty}(G)$ at the unit $\epsilon$. Thus $\left(\mathcal{G}^{*},[,]_{*}\right)$ is a Lie algebra called the dual of $(G, \pi)$ (or the dual of $\mathcal{G}$ ). While the double of $(G, \pi)$ is the vector space $\mathcal{G} \oplus \mathcal{G}^{*}$ equipped with the Lie bracket $[(x, \alpha),(y, \beta)]:=\left([x, y]_{\mathcal{G}}+a d_{\alpha}^{*} y-a d_{\beta}^{*} x,[\alpha, \beta]_{*}+a d_{x}^{*} \beta-a d_{y}^{*} \alpha\right)$. Here both coadjoint actions of $\mathcal{G}$ on $\mathcal{G}^{*}$ and $\left(\mathcal{G}^{*},[,]_{*}\right)$ on $\mathcal{G}$, are considered (see [1] $)$.

Denoting $r^{+}$a left invariant bivector field in $G$, whose value at the unit is $r_{\epsilon}^{+}=: r$ and $\left[r^{+}, r^{+}\right]$the Schouten bracket of $r^{+}$and itself, the equation

$$
r \in \wedge^{2} \mathcal{G}, \quad[r, r]:=\left[r^{+}, r^{+}\right]_{\epsilon}=0
$$

is called the Classical Yang Baxter Equation $(\mathrm{CYBE})$ in $\operatorname{Lie}(G)=: \mathcal{G}$, (see [11]). For a solution $r$ of the CYBE, the tensor $\pi:=r^{+}-r^{-}$defines a Poisson Lie structure in $G$ (see also [20], [25], ...).

We'll identify $\otimes^{2} \mathcal{G}$ and the space of linear maps $\mathcal{G}^{*} \rightarrow \mathcal{G}$ and will denote an element $r$ of $\otimes^{2} \mathcal{G}$ and the corresponding linear map $\alpha \mapsto r^{\sharp}(\alpha):=r(\alpha,$.$) by the same symbol. With such identifications, the 3$-vector $[r, r]$ above is given by $[r, r](\alpha, \beta, \gamma):=<[r(\alpha), r(\beta)], \gamma>+<[r(\beta), r(\gamma)], \alpha>+<[r(\gamma), r(\alpha)], \beta>$ for every $\alpha, \beta, \gamma \in \mathcal{G}^{*}$, where [,] is the Lie bracket in $\mathcal{G}$ and $\langle X, \alpha\rangle:=\alpha(X)$ is the usual canonical dual pairing between vectors and linear forms on $\mathcal{G}$. 
Definition 1.0.1. A Lie algebra $\mathcal{G}$ will be termed a Manin algebra if it has a (non definite) scalar product $<,>$ such that the following conditions hold.

(i) $<[x, y], z>+<y,[x, z]>=0, \forall x, y, z \in \mathcal{G}$, where [,] is the Lie bracket. The pair $(\mathcal{G},<,>)$ is also said to be an orthogonal Lie algebra.

(ii) $\mathcal{G}=A \oplus B$ possesses two supplementary totally isotropic subalgebras $A$ and $B$ of same dimension $\operatorname{dim}(A)=\operatorname{dim}(B)$. Vector spaces with this property are sometimes called hyperbolic.

Definition 1.0.2. A Left Symmetric Algebra (LSA) structure on a vector space $V$ is a product $V \times V \rightarrow$ $V,(x, y) \mapsto x y$ whose associator $a(x, y, z):=(x y) z-x(y z)$ is left symmetric i.e $a(x, y, z)=a(y, x, z)$, $\forall x, y, z \in V$.

A symplectic Lie group $\left(G, \omega^{+}\right)$, in the sense of Lichnerowicz-Medina [17], is a Lie group $G$ together with a left invariant symplectic form $\omega^{+}$. If $\mathcal{G}:=\operatorname{Lie}(G)$ and $\omega_{\epsilon}^{+}=: \omega$, then $(\mathcal{G}, \omega)$ is called a symplectic Lie algebra.

An LSA gives rise to a Lie bracket [,] on $V$ by $[x, y]:=x y-y x$. The Lie backet [,] is also said to be underlying the LSA structure. Any connected Lie group with Lie algebra $V_{-}:=(V,[]$,$) possesses a left$ invariant affine structure with associated connection $\nabla_{x^{+}} y^{+}:=(x y)^{+}$. The formula $\nabla_{x^{+}} y_{\epsilon}^{+}=: x y$ gives the converse way to get LSAs on Lie algebras from left invariant affine structures on Lie groups.

In the case of symplectic Lie groups, the connection defined by (1) induces a Left Symmetric Algebra (LSA) structure $(x, y) \rightarrow x y$ in $\mathcal{G}$, which is compatible with the Lie bracket of $\mathcal{G}$, ie $x y-y x=[x, y]$, $\forall x, y \in \mathcal{G}$. The formula (1) above, equips $G$ with a left invariant affine structure. In the Lie algebra level, $\omega^{+}$being closed reads

$$
\omega([x, y], z)+\omega([y, z], x)+\omega([z, x], y)=0, \quad \forall x, y, z \in \mathcal{G}
$$

On this work, a solution of the CYBE is always supposed to be skew-symmetric. A left invariant affine structure will always mean a (locally) flat (and torsion free) left invariant affine structure.

From now on, the scalar product $<,>$ stands for the duality scalar product (dual pairing) between the considered vector spaces and their duals.

\section{The CYBE and Affine Geometry}

Here is a simple remark about the Classical Yang Baxter Equation (CYBE), with several interesting consequences. Denote $Z(\mathcal{G})$ the center of a Lie algebra $\mathcal{G}$.

Lemma 2.0.1. Let $(G, \pi)$ be an exact Poisson Lie group, i.e $\pi:=r^{+}-r^{-}$, where $r \in \wedge^{2} \mathcal{G}$. Denote $\left(\mathcal{G}^{*},[,]_{*}\right)$ the dual Lie algebra of $(G, \pi)$. If for every $\alpha, \beta$ in $\mathcal{G}^{*}$ the following assumption holds

$r\left([\alpha, \beta]_{*}\right)-[r(\alpha), r(\beta)] \in Z(\mathcal{G})$

then any Lie group $G^{*}$ with Lie algebra $\left(\mathcal{G}^{*},[,]_{*}\right)$, is endowed with a left invariant affine connection given by $\quad \nabla_{\alpha^{+}} \beta^{+}:=\left(a d_{r(\alpha)}^{*} \beta\right)^{+}$.

The corresponding LSA structure on $\left(\mathcal{G}^{*},[,]_{*}\right)$ is given by $\alpha, \beta$ in $\mathcal{G}^{*}$

$$
\alpha \beta:=a d_{r(\alpha)}^{*} \beta .
$$

Proof. It suffices to check that the map

$$
\eta: \mathcal{G}^{*} \rightarrow \operatorname{aff}\left(\mathcal{G}^{*}\right), \alpha \mapsto\left(\alpha, a d_{r(\alpha)}^{*}\right)
$$

is a Lie algebra homomorphism, or equivalently to check the identity

$$
\left([\alpha, \beta]_{*}, a d_{r\left([\alpha, \beta]_{*}\right)}^{*}\right)=\left(a d_{r(\alpha)}^{*} \beta-a d_{r(\beta)}^{*} \alpha, a d_{[r(\alpha), r(\beta)]}^{*}\right) .
$$

But thanks to $(*)$ and the fact that one has

$$
[\alpha, \beta]_{*}=[\alpha, \beta]_{r}:=a d_{r(\alpha)}^{*} \beta-a d_{r(\beta)}^{*} \alpha,
$$

by definition of the Lie bracket on the dual of $(G, \pi)$, formula (7) is immediate. 
Corollary 2.0.1. Every Lie group is endowed with a left invariant affine structure, if it is dual to a Poisson Lie-group $(G, \pi)$, where $\pi=r^{+}-r^{-}$is given by a solution $r$ of the CYBE .

Corollary 2.0.2. If $G^{*}$ is a dual Lie group of a Poisson Lie group $(G, \pi)$, where $\pi=r^{+}-r^{-}$is given by a solution $r$ of the CYBE, then the derived Lie group $\left[G^{*}, G^{*}\right]$ of $G^{*}$ is not dense in $G^{*}$.

We can now state the following result.

Theorem 2.0.1. Let $G$ be a Lie group with (trivialized) cotangent bundle $T^{*} G=\mathcal{G}^{*} \rtimes G$, considered as a Lie group (semi-direct product of $G$ and the abelian Lie group $\mathcal{G}^{*}$, where $G$ acts on $\mathcal{G}^{*}$ by the coadjoint action). Then the solutions of the $C Y B E$ in $\mathcal{G}:=\operatorname{Lie}(G)$ are in one-to-one correspondence with the connected Lie subgroups of $T^{*} G$ equipped with left invariant affine structures whose Lie algebras are lagrangian graphs of a $\mathcal{G}$-valued skew-symmetric linear mapping of $\mathcal{G}^{*}$. Here the Lie algebra $t^{*} \mathcal{G}:=\mathcal{G}^{*} \rtimes \mathcal{G}$ of $T^{*} G$, is endowed with its canonical dual pairing $\langle(\alpha, x),(\beta, y)>:=\alpha(y)+\beta(x)$ for all $x, y \in \mathcal{G}$, $\alpha, \beta \in \mathcal{G}^{*}$.

Proof. Let $r$ be a solution of the CYBE on $\mathcal{G}$. Let's consider the bi-invariant metric (i.e canonical orthogonal structure) on $T^{*} G$

$<(\alpha, x)^{+},(\beta, y)^{+}>:=\alpha(y)+\beta(x)$.

One realizes that the map $\theta: \mathcal{D}(r) \rightarrow t^{*} \mathcal{G}:=\operatorname{Lie}\left(T^{*} G\right) ; \theta(\alpha, x):=(\alpha, r(\alpha)+x)$ is a Manin Lie algebras isomorphism (where $\mathcal{D}(r)$ is the double Lie algebra of $\left(G, r^{+}-r^{-}\right)$), see proposition 3.0.1. On the other hand, the Lie subalgebra $\mathcal{G}^{*}$ of $\mathcal{D}(r)$, dual to $\mathcal{G}$, is equipped with an LSA structure compatible with the Lie bracket of $\left(\mathcal{G}^{*},[,]_{r}\right)=: \mathcal{G}^{*}(r)$ and defined by $\alpha \beta=a d_{r(\alpha)}^{*} \beta$. Furthermore, the image of $\mathcal{G}^{*}(r)$ by $\theta$ is the graph of $r$. Thus, the connected Lie subgroup of $T^{*} G$, whose Lie algebra is $\theta\left(\mathcal{G}^{*}(r)\right)=\operatorname{graph}(r)$, is indeed endowed with a left invariant affine structure.

Conversely, let $K$ be a connected Lie subgroup of $T^{*} G$ satisfying the conditions of our statement, in particular $\operatorname{Lie}(K)=\operatorname{graph}(r)$, where $r: \mathcal{G}^{*} \rightarrow \mathcal{G}$ is skew symmetric. The Lie bracket in $\operatorname{graph}(r)$ is of the form

$$
\left[(\alpha, r(\alpha),(\beta, r(\beta))]=\left([\alpha, \beta]_{r},[r(\alpha), r(\beta)]\right)\right.
$$

where $[\alpha, \beta]_{r}=[\alpha, \beta]_{*}=a d_{r(\alpha)}^{*} \beta-a d_{r(\beta)}^{*} \alpha$.

The fact that $\operatorname{graph}(r)$ is a subalgebra of $t^{*} \mathcal{G}$ is equivalent to the following

$r\left([\alpha, \beta]_{r}\right)=[r(\alpha), r(\beta)]_{\mathcal{G}}$ for all $\alpha, \beta \in \mathcal{G}^{*}$.

But such an identity (with $r$ skew symmetric, $\operatorname{graph}(r)$ being totally isotropic) means that one has $[r, r]=0$. In fact, we have $[r, r](\alpha, \beta, \gamma)=<[r(\alpha), r(\beta)]-r\left([\alpha, \beta]_{r}\right), \gamma>$, for all $\alpha, \beta, \gamma \in \mathcal{G}^{*}$.

Let $G$ be a connected Lie group with Lie algebra $\mathcal{G}$, and $r \in \wedge^{2} \mathcal{G}$ a solution of the CYBE on $\mathcal{G}$. Now, we would like to study the geodesic completness of the left invariant affine structure induced by $r$ on every Lie group $G^{*}$ whose Lie algebra is the dual Lie algebra $\left(\mathcal{G}^{*},[,]_{r}\right)$ of $\left(G, r^{+}-r^{-}\right)$. Such a completness occurs if and only if the universal cover $\tilde{G}^{*}$ of $G^{*}$ is affinely diffeomorphic to the affine space $\mathbb{R}^{n}$, via a developping map. Equivalently, this means that in the LSA $\mathcal{G}^{*}$, all the right multiplications have a null trace.

Consider the left invariant Poisson tensor $r^{+}$on $G$ satisfying $r_{\epsilon}^{+}=r$. It's well known that the leaf through $\epsilon$, of the symplectic foliation of $r^{+}$, is a symplectic Lie group, whose Lie algebra is the image $\operatorname{Im}(r)$ of $r: \mathcal{G}^{*} \rightarrow \mathcal{G}$. The natural projection $p: \mathcal{G}^{*} \rightarrow \mathcal{G}^{*} / k e r(r) \simeq \operatorname{Im}(r)$ is a morphism of LSAs.

The symplectic form $\bar{\omega}$ in $\operatorname{Im}(r)$ is given by

$\bar{\omega}(r(\alpha), r(\beta)):=<\alpha, r(\beta)>, \forall \alpha, \beta \in \mathcal{G}^{*}$,

while the LSA structure in $\operatorname{Im}(r)$ is defined as follows

$\bar{\omega}(r(\alpha) r(\beta), r(\gamma)):=<a d_{r(\alpha)}^{*} \beta, r(\gamma)>$ or equivalently $r(\alpha) r(\beta):=r\left(a d_{r(\alpha)}^{*} \beta\right)$.

Denoting by $R_{\alpha}$ (resp. $R_{\alpha}^{i m}$ ) the right multiplication by $\alpha$ (resp. $\left.r(\alpha)\right)$ in $\mathcal{G}^{*}$ (resp. in $\operatorname{Im}(r)$ ), one can show that $R_{\alpha}$ and $R_{r(\alpha)}^{i m}$ have the same trace. We then deduce the following important result.

Theorem 2.0.2. (Geodesic completness of the affine structure) Let $G^{*}$ be a Lie group, with Lie algebra $\left(\mathcal{G}^{*},[,]_{r}\right)$. The left invariant affine stucture on $G^{*}$, induced by $r$, is complete if and only if the leaf $\mathcal{F}_{\epsilon}$ through $\epsilon$, of the symplectic foliation of $r^{+}$is a unimodular Lie group. So completness implies the solvability of $G^{*}$. Here $r^{+}$stands for the left invariant Poisson tensor whose value at $\epsilon$ is $r$. 
Equivalently, we also have

Theorem 2.0.2 bis.

The left invariant affine stucture on $G^{*}$, induced by $r$, is complete if and only if the quotient $\mathcal{G}^{*} / K e r(r)$, is a unimodular and solvable Lie algebra. In this case $G^{*}$ is solvable, as $\operatorname{Ker}(r)$ and $\mathcal{G}^{*} / \operatorname{Ker}(r)$ are. Here $\operatorname{Ker}(r)$ is the kernel of the skew symmetric linear map $r: \mathcal{G}^{*} \rightarrow \mathcal{G} ; \alpha \mapsto r(\alpha,)=.: i_{\alpha} r$.

Remark that $\operatorname{Ker}(r)$ is an abelian two-sided ideal of the LSA $\mathcal{G}^{*}$.

\section{The double of a symplectic Lie group is affine and complex}

Denote $\mathcal{D}(\mathcal{G})$ the double Lie algebra of a Poisson Lie group $(G, \pi)$, where $\pi:=r^{+}-r^{-}$is given by a solution $r$ of the CYBE on $\mathcal{G}:=\operatorname{Lie}(G)$. Let's point out the following interesting result, whose proof is immediate.

Proposition 3.0.1. The mapping $\Xi:(x, \alpha) \mapsto(x+r(\alpha), \alpha)$ is a Lie algebra isomorphism and an isometry between the Manin algebras $\mathcal{D}(\mathcal{G})$ and $\mathcal{G} \ltimes_{a d^{*}} \mathcal{G}^{*}$.

Now, let $(\mathcal{G}, w)$ be a symplectic Lie algebra. Consider the vector space isomorphism

$$
\begin{aligned}
q: \mathcal{G} & \rightarrow \mathcal{G}^{*} \\
x & \mapsto q(x)
\end{aligned}
$$

where $\langle q(x), y\rangle=w(x, y)$ for all $x, y$ in $\mathcal{G}$ and $<,>$ is the duality scalar product.

Recall that $r:=q^{-1}$ regarded as an element of $\wedge^{2} \mathcal{G}$, is a solution of the CYBE on $\mathcal{G}$. Conversely associated to an invertible solution $r$ of the CYBE on $\mathcal{G}$, is a symplectic Lie algebra $(\mathcal{G}, \omega)$ by setting $\omega(x, y):=<r^{-1}(x), y>$.

Now using $q$, let's transport the LSA and the Lie algebra structures of $\mathcal{G}$ to the vector space $\mathcal{G}^{*}$, in such a way as $q$ becomes an isomorphism of LSAs. One then obtains compatible Lie algebra and LSA structures on $\mathcal{G}^{*}$. They are respectively given by

$$
\begin{gathered}
{[\alpha, \beta]_{q}:=q([r(\alpha), r(\beta)])} \\
\alpha \beta:=q(r(\alpha) r(\beta))
\end{gathered}
$$

for all $\alpha, \beta \in \mathcal{G}^{*}$, where $r(\alpha) r(\beta)$ is the product of $r(\alpha)$ and $r(\beta)$ in $\mathcal{G}$, induced by (1).

The following can be proved by some direct computations.

Lemma 3.0.2. For every $x, y \in \mathcal{G}$ and $\alpha, \beta \in \mathcal{G}^{*}$ one has

$$
\begin{aligned}
& \text { (a) } a d_{x}^{*} q(y)=q(x y)=: q(x) q(y) \quad \text { or equivalently } q \circ l_{x}=a d_{x}^{*} \circ q \\
& \left(a^{\prime}\right) \quad r\left(a d_{x}^{*} \beta\right)=x r(\beta)=\left(l_{x} \circ r\right)(\beta), \quad r \circ a d_{x}^{*}=\left(l_{x} \circ r\right) \\
& (b) \quad r\left(a d_{x}^{*} \beta\right)=a d_{q(x)}^{*} r(\beta) \\
& (c) \quad[\alpha, \beta]_{q}=a d_{r(\alpha)}^{*} \beta-a d_{r(\beta)}^{*} \alpha \\
& (d) \quad q(x) \beta=a d_{x}^{*} \beta \\
& (e) \quad r(\beta) x=a d_{\beta}^{*} x
\end{aligned}
$$

\section{Remarks 3.0.1.}

The formula (d) means that the LSA structure on $\mathcal{G}^{*}$ defined by the formula (5) coincides with the one given in (10), which is the image by $q$ of (1]).

Thanks to (c), the Lie bracket $[,]_{q}$ is the one underlying the LSA structure on $\mathcal{G}^{*}$ given by (5), and coincides with the one (namely $\left.[,]_{r}\right)$ defined by (8). 
Let's consider the coadjoint representations of $\mathcal{G}$ and $\left(\mathcal{G}^{*},[,]_{q}\right)$ respectively on $\mathcal{G}^{*}$ and $\mathcal{G}$. On the vector space $\mathcal{D}:=\mathcal{G} \oplus \mathcal{G}^{*}$, one defines the product: $x, y \in \mathcal{G}$ and $\alpha, \beta \in \mathcal{G}^{*}$

$$
(x, \alpha)(y, \beta):=\left(x y+a d_{\alpha}^{*} y, \alpha \beta+a d_{x}^{*} \beta\right)
$$

We can now state the main result of this paragraph, whose proof is given later.

Theorem 3.0.3. Let $r \in \wedge^{2} \mathcal{G}$ be an invertible solution of the CYBE on a Lie group $G$ with Lie algebra $\mathcal{G}$. Then every Lie group is endowed with a left invariant affine structure if its Lie algebra is isomorphic to the double Lie algebra $\mathcal{D}=\mathcal{G} \oplus \mathcal{G}^{*}$ of the Poisson Lie group $\left(G, \pi:=r^{+}-r^{-}\right)$. The associated LSA in $\mathcal{D}$ is given by the formula (11).

Let $\left(G, \omega^{+}\right)$be a symplectic Lie group. Consider the left invariant affine connection $\nabla$ in $G$ and the corresponding LSA structure on $\mathcal{G}$.

From theorem 3.0.3 and proposition 3.0.1 one deduces the

Corollary 3.0.3. Let $\left(G, \omega^{+}\right)$be a symplectic Lie group, with symplectic Lie algebra $(\mathcal{G}, \omega)$.

The map $\Xi$ of proposition $\mathbf{3 . 0 . 1}$ gives rise to a left invariant affine (i.e flat and torsion free) connection $\tilde{\nabla}$ on the cotangent bundle $T^{*} G$ of $G$, in such a way that the natural fiber map (projection) is a morphism between the affine manifolds $\left(T^{*} G, \tilde{\nabla}\right)$ and $(G, \nabla)$. The LSA structure induced by $\tilde{\nabla}$ on Lie $\left(T^{*} G\right)=$ $\mathcal{G} \ltimes_{a d^{*}} \mathcal{G}^{*}$ is given by $(x, \alpha)(y, \beta):=\left(x y, a d_{x}^{*} \beta\right)$, where $x y$ is the product of $x$ and $y$ on $\mathcal{G}$, induced by (1): $\omega(x y, z):=-\omega(y,[x, z]), \forall z \in \mathcal{G}$.

Below, we give a method to build algebras with LSA structure, that generalizes the theorem 3.0.3.

Let $A$ and $B$ be two LSAs, denote $A_{-}$et $B_{-}$the respective underlying Lie algebras.

Definition 3.0.3. $A$ and $B$ are $\left(\theta_{1}, \theta_{2}\right)$-linked if there exist two Lie algebra representations

$$
\theta_{1}: A_{-} \rightarrow \mathcal{G l}(B) \quad \text { and } \quad \theta_{2}: B_{-} \rightarrow \mathcal{G l}(A)
$$

such that

$$
\begin{gathered}
\left(\theta_{1}(a) b\right) b^{\prime}+b\left(\theta_{1}(a) b^{\prime}\right)-\theta_{1}(a)\left(b b^{\prime}\right)=\theta_{1}\left(\theta_{2}(b) a\right) b^{\prime} \quad \text { and } \\
\left(\theta_{2}(b) a\right) a^{\prime}+a\left(\theta_{2}(b) a^{\prime}\right)-\theta_{2}(b)\left(a a^{\prime}\right)=\theta_{2}\left(\theta_{1}(a) b\right) a^{\prime}
\end{gathered}
$$

for all elements $a, a^{\prime}$ in $A$ and $b, b^{\prime}$ in $B$.

Lemma 3.0.3. I. Let $A$ and $B$ be two $\left(\theta_{1}, \theta_{2}\right)$-linked $L S A$ s, then the vector space $V=A \times B$ is endowed with an LSA structure given by the product of every two elements $(a, b)$ and $\left(a^{\prime}, b^{\prime}\right)$ of $A \times B$ :

$$
(a, b)\left(a^{\prime}, b^{\prime}\right)=\left(a a^{\prime}+\theta_{2}(b) a^{\prime}, b b^{\prime}+\theta_{1}(a) b^{\prime}\right)
$$

with underlying Lie bracket:

$$
\left[(a, b),\left(a^{\prime}, b^{\prime}\right)\right]=\left(\left[a, a^{\prime}\right]+\theta_{2}(b) a^{\prime}-\theta_{2}\left(b^{\prime}\right) a,\left[b, b^{\prime}\right]+\theta_{1}(a) b^{\prime}-\theta_{1}\left(a^{\prime}\right) b\right)
$$

II. Conversely every LSA with two supplementary left ideals, is built with the above method.

Before giving the proof of this lemma, let's remark an interesting example of LSA that can be obtained by such a construction. The LSA corresponding to the so-called flat left-invariant connections adapted to the automorphism structure of a Lie group, are obtained in this way, as they arise in [21] to be a direct sum of two ideals (see $[21]$ for more details).

Proof of lemma 3.0.3. The bilinearity is obvious. To prove that (14) defines an LSA structure on $V$, it suffices, identifying an element $a$ of $A$ (resp. $b$ of $B$ ) with the element $(a, 0)(\operatorname{resp} .(0, b))$ of $A \times B$; to check that:

(i) $\quad(a b) b^{\prime}-a\left(b b^{\prime}\right)=(b a) b^{\prime}-b\left(a b^{\prime}\right)$

(ii) $\quad(b a) a^{\prime}-b\left(a a^{\prime}\right)=(a b) a^{\prime}-a\left(b a^{\prime}\right)$

(iii) $\quad\left(b b^{\prime}\right) a-b\left(b^{\prime} a\right)=\left(b^{\prime} b\right) a-b^{\prime}(b a)$

(iv) $\quad\left(a a^{\prime}\right) b-a\left(a^{\prime} b\right)=\left(a^{\prime} a\right) b-a^{\prime}(a b)$ 
Then, one checks that

(i) is equivalent to the hypothesis (12)

(ii) is equivalent to the hypothesis (13)

(iii) expresses the fact $\theta_{2}$ is a representation of the Lie algebra $B_{-}$and

(iv) expresses the fact $\theta_{1}$ is a representation of the Lie algebra $A_{-}$.

Conversely, let's suppose that $A$ and $B$ are two left (sided) ideals of an LSA $\mathcal{G}=A \oplus B$. The maps defined by $\theta_{1}(a) b:=a b$ and $\theta_{2}(b) a:=b a$ for all $a \in A$ and all $b \in B$ satisfy the conditions I of this lemma and then enable us to construct an LSA structure on $\mathcal{G}$ which coincides with the initial one.

\subsubsection{Proof of theorem 3.0.3}

- Proof of the first assertion.

In the lemma 3.0.3, put $A=\mathcal{G}, B=\mathcal{G}^{*}$ and denote the coadjoint representations of $\mathcal{G}$ and $\mathcal{G}^{*}$ respectively by $\theta_{1}$ and $\theta_{2}$.

For $x \in \mathcal{G}, \alpha$ and $\beta$ in $\mathcal{G}^{*}$ let's consider the following

$\Theta=\left(\theta_{1}(x) \alpha\right) \beta+\alpha\left(\theta_{1}(x) \beta\right)-\theta_{1}(x)(\alpha \beta)$

Using the formulas (a)-(e) and the fact that the product defines an LSA structure, one has

$$
\begin{aligned}
\Theta & =\left(a d_{x}^{*} \alpha\right) \beta+\alpha\left(a d_{x}^{*} \beta\right)-a d_{x}^{*}(\alpha \beta) \\
& =q(x r(\alpha)) q(r(\beta))+q(r(\alpha)) q(x r(\beta))-q(x(r(\alpha) r(\beta))) \\
& =q((x r(\alpha)) r(\beta)+r(\alpha)(x r(\beta))-x(r(\alpha) r(\beta))) \\
& =q(((x r(\alpha)) r(\beta)-x(r(\alpha) r(\beta)))+r(\alpha)(x r(\beta))) \\
& =q((r(\alpha) x) r(\beta)-r(\alpha)(x r(\beta))+r(\alpha)(x r(\beta))) \\
& =q((r(\alpha) x) r(\beta)) \\
& =q\left(a d_{\alpha}^{*} x\right) \beta=a d_{a d_{\alpha}^{*} x}^{*} \beta \\
& =\theta_{1}\left(\theta_{2}(\alpha) x\right) \beta
\end{aligned}
$$

The first hypothesis of lemma 3.0 .3 is then satisfied. The second one is exactly checked in the same way. Then, lemma $\mathbf{3 . 0 . 3}$ allows us to make a conclusion to the first statement of this theorem.

- The second statement is directly deduced from both remarks $\mathbf{3 . 0 . 1}$ and lemma $\mathbf{3 . 0 . 3}$.

Let $x$ and $\beta$ respectively be elements of the left sided ideals $\mathcal{G}$ and $\mathcal{G}^{*}$ of $\mathcal{D}(\mathcal{G})$. We can show that the trace of the right multiplication by $x$ in $\mathcal{D}(\mathcal{G})$ is exactly equal to the one of the right multiplication by $x$ in $\mathcal{G}$, while the trace of the right multiplication by $\alpha$ in $\mathcal{D}(\mathcal{G})$ is exactly equal to the one of the right multiplication by $r(\alpha)$ in $\mathcal{G}$. Finally we claim

\section{Theorem 3.0.4. (Geodesic completness of the double)}

Let $G$ be connected a Lie group with Lie algebra $\mathcal{G}$ and $r \in \wedge^{2} \mathcal{G}$ an invertible solution of the CYBE. Consider the LSA structure in the double Lie algebra $\mathcal{D}(\mathcal{G})$ of $\left(G, r^{+}-r^{-}\right)$as described in the theorem 3.0.3. The following are equivalent:

1. on every connected Lie group $D(\mathcal{G})$ whose Lie algebra is $\mathcal{D}(\mathcal{G})$; the left invariant affine structure corresponding to the LSA, is geodesically complete.

2. the Lie group $G$ is unimodular (and solvable).

Let's denote again by $\mathcal{D}(\mathcal{G})$ the double Lie algebra of a symplectic Lie group $\left(G, \omega^{+}\right)$, and by $q$ as above. One has

Proposition 3.0.2. Every Lie group, whose Lie algebra is the double Lie algebra $\mathcal{D}(\mathcal{G})$ of $(G, w)$, is endowed with left invariant complex structure defined by the endomorphism

$$
J(x, \alpha) \mapsto\left(-q^{-1}(\alpha), q(x)\right)
$$

of $\mathcal{D}(\mathcal{G})$; where $q(x):=\omega(x,$.$) .$

To prove the above proposition, one just checks that the Nijenhuis tensor

$N_{J}\left(v_{1}, v_{2}\right)=\left[J v_{1}, J v_{2}\right]-\left[v_{1}, v_{2}\right]-J\left[v_{1}, J v_{2}\right]-J\left[J v_{1}, v_{2}\right]$

of $J$, vanishes for all $v_{1}=(x, \alpha), v_{2}=(y, \beta) \in \mathcal{D}(\mathcal{G})$. 


\section{The Poisson-Lie structure associated to a symplectic Lie-group $\left(G, \omega^{+}\right)$is polynomial of degree 2}

Let $\left(G, \omega^{+}\right)$be a symplectic Lie group, $\epsilon$ its unit and $(\mathcal{G}, \omega)$ its symplectic Lie algebra, where $\omega=\omega_{\epsilon}^{+}$.

Consider, as in section 3 the vector spaces (skew-symmetric) isomorphism

$$
\begin{aligned}
q: \mathcal{G} & \rightarrow \mathcal{G}^{*} \\
x & \mapsto q(x):=i_{x} \omega=\omega(x, .)
\end{aligned}
$$

Due to the relation $\partial \omega=0$, ie $\omega^{+}$is closed, $q$ is actually a 1-cocycle for the coadjoint representation of $\mathcal{G}$. Again, denote $r=q^{-1}$ the inverse of $q$, considered when needed, as an element of $\wedge^{2} \mathcal{G}$ as well.

Let $\tilde{G}$ be the connected and simply connected Lie group with Lie algebra $\mathcal{G}$. The invertible 1-cocycle $q$ can be integrated into a 1-cocycle $Q: \tilde{G} \rightarrow \mathcal{G}^{*}$ for the coadjoint representation of $\tilde{G}$. Such a 1-cocycle $Q$ is a cover over the open subset $\operatorname{Im} Q$ of $\mathcal{G}^{*}$, and is equivariant relative to the actions of $\tilde{G}$ on itself by left multiplications, and on $\mathcal{G}^{*}$ by the affine representation

$$
\begin{array}{cl}
\tilde{G} & \stackrel{\rho}{\rightarrow} \operatorname{Aff}\left(\mathcal{G}^{*}\right)=\mathcal{G}^{*} \rtimes G L\left(\mathcal{G}^{*}\right) \\
\sigma & \mapsto \rho(\sigma)=\left(Q(\sigma), A d_{\sigma}^{*}\right)
\end{array}
$$

Namely, $Q$ is given by the expression

$$
Q(\tau)=\sum_{k=1}^{k=+\infty} \frac{\left(a d_{a}^{*}\right)^{k-1}}{k !} q(a)
$$

where $\tau=\exp (a)$ and $a$ is in $\mathcal{G}$.

The affine representation $\rho$ then has an open orbit $\operatorname{Orbit}(0)=\operatorname{Im} Q$, here 0 stands for the origine of the vector space $\mathcal{G}^{*}$. Pulling back (using $Q$ ) the usual affine structure on the open subset $\operatorname{Orbit}(0)$ of $\mathcal{G}^{*}$, one obtains a left invariant affine structure on $\tilde{G}$. Actually, $Q$ is a developping map of such a structure on $\tilde{G}$.

Lemma 4.0.4. There exists a left invariant affine structure on $G$ such that the "universal covering map" $\tilde{G} \stackrel{p}{\rightarrow} G$ is a morphism of affine manifolds. Such an affine structure coincides with the one given by $\omega^{+}$ on $G$. Furthermore $p$ is Poisson morphism between the Poisson Lie-groups $\left(\tilde{G}, r^{+}-r^{+}\right)$and $\left(G, r^{+}-r^{+}\right)$.

\section{Proof.}

As the group of deck transformations $\pi_{1}(G)$ is a central and discrete subgroup of $\tilde{G}$, it properly and freely acts on $\tilde{G}$ by left translations which are affine transformations. Consequently, $G=\tilde{G} / \pi_{1}(G)$ is equipped with a left invariant affine structure relative to which, $p$ is a morphism of affine manifolds. Realizing that $q$ is the orbital map (for the infinitesimal action) at 0 , we deduce the coincidence between such an affine structure on $G$ and the one given by $\omega^{+}$.

Let's denote $\tilde{\pi}(\operatorname{resp} . \pi)$, the tensor on $\tilde{G}(\operatorname{resp} . G)$ given by $r$. Let $\tilde{\epsilon}$ be the unit of $\tilde{G}$ and $\tilde{L}_{\sigma}$ (resp. $\left.\tilde{R}_{\sigma}\right)$ be the left (resp. right) translation in $\tilde{G}$ by $\sigma \in \tilde{G}$. Then, for every $\sigma \in \tilde{G}$, one has

$T_{\sigma} p \tilde{\pi}_{\sigma}=T_{\sigma} p\left(T_{\tilde{\epsilon}} \tilde{L}_{\sigma} r\right)-T_{\sigma} p\left(T_{\tilde{\epsilon}} \tilde{R}_{\sigma} r\right)=T_{\tilde{\epsilon}}\left(p \circ \tilde{L}_{\sigma}\right) r-T_{\tilde{\epsilon}}\left(p \circ \tilde{R}_{\sigma}\right) r=\left(T_{\epsilon} L_{p(\sigma)}\right) r-\left(T_{\epsilon} R_{p(\sigma)}\right) r=: \pi_{p(\sigma)}$.

Then, $p$ is a Poisson morphism between $(\tilde{G}, \tilde{\pi})$ and $(G, \pi)$.

Remarks 4.0.2. 1. As the covering map $p$ is a morphism of both affine and Poisson manifolds, to proof that $\pi$ is polynomial, it suffices to prove that $\tilde{\pi}$ is polynomial.

2. It's convenient to point out that for every $\gamma \in \pi_{1}(G)$, the following diagram commutates

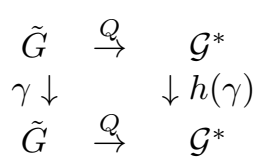

Here $h: \pi_{1}(G) \rightarrow \operatorname{Aff}\left(\mathcal{G}^{*}\right)$ is the Lie groups homomorphism, the so called holonomy representation of the affine manifold $G$.

3. One easily verifies that $Q$ is a moment map for the action of $\tilde{G}$ on itself, via left multiplications. 
Here is our main result of this section. It states that for every invertible solution $r$ of the CYBE, the corresponding Poisson Lie structure $\pi:=r^{+}-r^{-}$is polynomial, of degree smaller or equal to 2 .

Theorem 4.0.5. Let $\left(G, \omega^{+}\right)$be a connected symplectic Lie group. Denote $r$ the associated solution of $C Y B E$ and $r^{+}$(resp. $r^{-}$) the corresponding left (resp. right) invariant Poisson tensor.

Then, the Poisson Lie tensor $\pi=r^{+}-r^{-}$is polynomial, of degree smaller or equal to 2 , relative to the left invariant affine structure on $G$ defined by $\omega^{+}$. That is, at the neighborhood of every point of $G$, there exists local affine coordinates $\left(x_{1}, \ldots, x_{2 n}\right)$ satisfying

$$
\left\{x_{i}, x_{j}\right\}_{\pi}=\sum_{s} b_{s} x_{s}+\sum_{l, k} b_{l k} x_{l} x_{k} \text { where the } b_{s}, b_{l k} \text { 's are in } \mathbb{R} \text {. }
$$

\subsection{Proof of theorem 4.0 .5}

To prove that $\pi$ is polynomial, thanks to remarks 4.0.2, it suffices to prove that $\tilde{\pi}$ is polynomial. We can then suppose, without any lost of generalities, that $G$ is connected and simply connected.

We will then prove that $\pi$ can be projected by $Q$ to a Poisson tensor on $\operatorname{Im} Q$ which is polynomial, of degree smaller or equal to 2 .

The following very useful two lemmas will be proved later.

Lemma 4.1.1. Let $\sigma$ be in $G$ and $T_{\sigma} Q$ be the differential of $Q$ at $\sigma$, the tensor $T_{\sigma} Q \pi_{\sigma}$ only depends on $Q(\sigma)$.

This means that if two elements $\sigma$ and $\sigma^{\prime}$ of $G$ have the same image $Q(\sigma)=Q\left(\sigma^{\prime}\right)$ by $Q$, then one has $T_{\sigma} Q \pi_{\sigma}=T_{\sigma^{\prime}} Q \pi_{\sigma^{\prime}}$.

The lemma 4.1.1 solves the main problem and then proves that the tensor $\pi$ can be projected, by $Q$, to a Poisson tensor $\Lambda=Q_{*} \pi$ on $\operatorname{Im} Q$.

Lemma 4.1.2. Let $f$ and $g$ be two elements of $\left(\mathcal{G}^{*}\right)^{*}=\mathcal{G}$, denote $\{f, g\}_{\Lambda}$ their Poisson brackets relative to $\Lambda$. Then one has

$$
\begin{aligned}
\{f, g\}_{\Lambda}(Q(\sigma)) & =-<Q(\sigma),[f, g]>-r\left(a d_{f}^{*} Q(\sigma), a d_{g}^{*} Q(\sigma)\right) \\
& =-<Q(\sigma),[f, g]>-<\left(a d_{f} \otimes a d_{g}\right) r, Q(\sigma) \otimes Q(\sigma)>
\end{aligned}
$$

Then, the bracket of the restriction to $\operatorname{Im} Q$ of two linear functions on $\mathcal{G}^{*}$, is a polynomial function of degree 2 , on $\operatorname{Im} Q$. Being understood that $[f, g]$ is a linear form on $\mathcal{G}^{*}$, the function

$$
\begin{aligned}
u: \operatorname{Im} Q & \rightarrow \mathbb{R} \\
Q(\sigma) & \mapsto u(Q(\sigma)):=r\left(a d_{f}^{*} Q(\sigma), a d_{g}^{*} Q(\sigma)\right)=<\left(a d_{f} \otimes a d_{g}\right) r, Q(\sigma) \otimes Q(\sigma)>
\end{aligned}
$$

is polynomial (with respect to the coordinates of $Q(\sigma)$ ) homogeneous of degree 2, and the element $\left(a d_{f} \otimes a d_{g}\right) r$ of $\otimes^{2} \mathcal{G}$ is a bilinear form on $\mathcal{G}^{*}$.

This ends the proof of the theorem.

\subsection{Proof of lemmas 4.1.1 et 4.1 .2}

\section{a. Proof of lemma 4.1.1}

Let $f$ and $g$ be in $\mathcal{C}^{\infty}(\operatorname{Im} Q)$; one has

$$
\begin{aligned}
T_{\sigma} Q \pi_{\sigma}(d f(Q(\sigma)), d g(Q(\sigma))) & =\pi_{\sigma}\left(d f(Q(\sigma)) \circ T_{\sigma} Q, d g(Q(\sigma)) \circ T_{\sigma} Q\right) \\
& =<\pi_{\sigma}^{\sharp}\left(d f(Q(\sigma)) \circ T_{\sigma} Q\right), d g(Q(\sigma)) \circ T_{\sigma} Q> \\
& =<\left(T_{\sigma} Q \circ \pi_{\sigma}^{\sharp} \circ\left(T_{\sigma} Q\right)^{t}\right)(d f(Q(\sigma)), d g(Q(\sigma))>
\end{aligned}
$$

Let's then prove that the expression $T_{\sigma} Q \circ \pi_{\sigma}^{\sharp} \circ\left(T_{\sigma} Q\right)^{t}$ only depends on $Q(\sigma)$. To do this, we will need the following formulas. Denote by $\Omega$ the differential 2-form $\Omega=\omega^{+}-\omega^{-}$and $\Omega^{b}$ the corresponding morphism of vector bundles:

$$
\begin{aligned}
& T_{\sigma} G \stackrel{\Omega^{b}}{\rightarrow} T_{\sigma}^{*} G \\
& X_{\sigma} \mapsto \Omega_{\sigma}^{b}\left(X_{\sigma}\right)
\end{aligned}
$$


where $<\Omega_{\sigma}^{b}\left(X_{\sigma}\right), Y_{\sigma}>:=\Omega_{\sigma}\left(X_{\sigma}, Y_{\sigma}\right)$ for all $X_{\sigma}, Y_{\sigma} \in T_{\sigma} G$.

Then for all $\sigma \in G$, the equality

$$
{ }^{t}\left(T_{\epsilon} L_{\sigma}\right) \circ \Omega_{\sigma}^{b} \circ T_{\epsilon} L_{\sigma}=q-A d^{*}\left(\sigma^{-1}\right) \circ q \circ \operatorname{Ad}(\sigma)
$$

is due to the following, satisfied by every $x$ and $y$ in $\mathcal{G}$ :

$$
\begin{aligned}
<\left(q-A d_{\sigma^{-1}}^{*} \circ q \circ A d_{\sigma}\right)(x), y> & =w(x, y)-w\left(A d_{\sigma} x, A d_{\sigma} y\right) \\
& =\omega_{\sigma}^{+}\left(x_{\sigma}^{+}, y_{\sigma}^{+}\right)-\omega_{\sigma}^{-}\left(x_{\sigma}^{+}, y_{\sigma}^{+}\right) \\
& =\Omega_{\sigma}\left(x_{\sigma}^{+}, y_{\sigma}^{+}\right)=<\Omega_{\sigma}^{b}\left(x_{\sigma}^{+}\right), y_{\sigma}^{+}> \\
& =<^{t}\left(T_{\epsilon} L_{\sigma}\right)\left(\Omega_{\sigma}^{b} \circ T_{\epsilon} L_{\sigma}\right)(x), y>.
\end{aligned}
$$

For $\sigma \in G$ the map $q^{\sigma}=A d_{\sigma^{-1}}^{*} \circ q \circ A d_{\sigma}$ is a 1-cocycle of $\mathcal{G}$. This is due to the fact that $A d_{\sigma}$ is a Lie algebra homomorphism and $q$ is a 1-cocycle of $\mathcal{G}$.

Let's integrate $q^{\sigma}$ into a 1-cocyle $Q^{\sigma}: G \rightarrow \mathcal{G}^{*}$, for the coadjoint action of $G$. Set $\tau=\exp (a)$, where $a$ is in $\mathcal{G}$. Then

$$
\begin{aligned}
Q^{\sigma}(\tau) & =\sum_{k=1}^{k=+\infty} \frac{\left(a d_{a}^{*}\right)^{k-1}}{k !}\left(A d_{\sigma^{-1}}^{*} \circ q \circ A d_{\sigma}\right)(a) \\
& =A d_{\sigma^{-1}}^{*} \sum_{k=1}^{k=+\infty} \frac{\left(a d_{\left(A d_{\sigma} a\right)}^{*}\right)^{k-1}}{k !}\left(q\left(A d_{\sigma} a\right)\right)
\end{aligned}
$$

as $a d_{a}^{*} \circ A d_{\sigma-1}^{*}$ is equal to $A d_{\sigma^{-1}}^{*} \circ a d_{A d_{\sigma} a}^{*}$, for every $a$ in $\mathcal{G}$ and $\sigma$ in $G$. The last assertion is due to the following

$$
\begin{aligned}
<a d_{a}^{*} \circ A d_{\sigma-1}^{*} \alpha, x> & =-<\alpha, A d_{\sigma}[a, x]>=-<\alpha,\left[A d_{\sigma} a, A d_{\sigma} x\right]> \\
& =<A d_{\sigma^{-1}}^{*} \circ a d_{A d_{\sigma} a}^{*} \alpha, x>
\end{aligned}
$$

for all $x$ in $\mathcal{G}$ and $\alpha$ in $\mathcal{G}^{*}$.

$$
\begin{aligned}
Q^{\sigma}(\tau) & =A d_{\sigma^{-1}}^{*}\left(Q\left(\exp \left(A d_{\sigma} a\right)\right)\right)=A d_{\sigma^{-1}}^{*} Q\left(\sigma \tau \sigma^{-1}\right) \\
& =A d_{\sigma-1}^{*}\left(Q(\sigma)+A d_{\sigma}^{*} Q\left(\tau \sigma^{-1}\right)\right) \\
& \left.=A d_{\sigma^{-1}}^{*} Q(\sigma)+Q(\tau)+A d_{\tau}^{*} Q\left(\sigma^{-1}\right)\right) \\
& \left.=-Q\left(\sigma^{-1}\right)+Q(\tau)+A d_{\tau}^{*} Q\left(\sigma^{-1}\right)\right)
\end{aligned}
$$

Because, one has

$\exp \left(A d_{\sigma} a\right)=\sigma \exp (a) \sigma^{-1}=\sigma \tau \sigma^{-1}$ and $Q\left(\sigma^{-1}\right)=-A d_{\sigma^{-1}}^{*} Q(\sigma)$.

We then obtain the equality

$\left.\left(Q-Q^{\sigma}\right)(\tau)=Q\left(\sigma^{-1}\right)-A d_{\tau}^{*} Q\left(\sigma^{-1}\right)\right)=-d\left(Q\left(\sigma^{-1}\right)\right)(\tau)$.

Here $d$ is the (Chevalley-Eilenberg) differential associated to the coadjoint representation of $G$ by $d \alpha(\sigma):=$ $A d_{\sigma}^{*} \alpha-\alpha$, if $\alpha \in \mathcal{G}^{*}$ and $\sigma \in G$.

In other words, $Q$ and $Q^{\sigma}$ are cohomologous and their difference is the 1-coboundary $Q-Q^{\sigma}=$ $-d\left(Q\left(\sigma^{-1}\right)\right)$. Differentiating at $\epsilon$ the relation (d), one establishes that $q$ and $q^{\sigma}$ are also cohomologous, and

$q-q^{\sigma}=-\delta\left(Q\left(\sigma^{-1}\right)\right)$

Here, $\delta$ is the differential associated to (infinitesimal) coadjoint action of $\mathcal{G}$ : if $x \in \mathcal{G}$ and $\alpha \in \mathcal{G}^{*}$ we have $\delta \alpha(x):=a d_{x}^{*} \alpha$.

The relation (b) above, then reads

$\Omega^{b}=-\left(T_{\sigma} L_{\sigma-1}\right)^{t} \circ \delta\left(Q\left(\sigma^{-1}\right)\right) \circ T_{\sigma} L_{\sigma-1}$

It is easy to check that one has

$$
\begin{aligned}
& \left(r^{+}\right)^{\sharp}=T_{\epsilon} L_{\sigma} \circ r \circ\left(T_{\epsilon} L_{\sigma}\right)^{t} ;\left(r^{-}\right)^{\sharp}=T_{\epsilon} R_{\sigma} \circ r \circ\left(T_{\epsilon} R_{\sigma}\right)^{t} \\
& q_{\sigma}^{+}:=\left(\omega^{+}\right)_{\sigma}^{b}=\left(T_{\sigma} L_{\sigma^{-1}}\right)^{t} \circ q \circ\left(T_{\sigma} L_{\sigma}^{-1}\right) ; \\
& q_{\sigma}^{-}:=\left(\omega^{-}\right)_{\sigma}^{b}=\left(T_{\sigma} R_{\sigma}^{-1}\right)^{t} \circ q \circ\left(T_{\sigma} R_{\sigma}^{-1}\right)
\end{aligned}
$$

We also have $T_{\sigma} Q=A d_{\sigma}^{*} \circ q \circ T_{\sigma} L_{\sigma-1}$,

as the following equalities are true, for every $x$ in $\mathcal{G}$

$$
\begin{aligned}
T_{\sigma} Q\left(x_{\sigma}^{+}\right) & =\left.\frac{d}{d t}\right|_{t=0} Q(\sigma \operatorname{exptx})=\left.\frac{d}{d t}\right|_{t=0}\left(Q(\sigma)+A d_{\sigma}^{*} Q(\operatorname{exptx})\right) \\
& =A d_{\sigma}^{*} q(x)=A d_{\sigma}^{*} \circ q \circ T_{\sigma} L_{\sigma^{-1}}\left(x_{\sigma}^{+}\right) .
\end{aligned}
$$


In such a way that we have

$$
\begin{aligned}
{ }^{t}\left(T_{\sigma} Q\right) & =\left(T_{\sigma} L_{\sigma^{-1}}\right)^{t} \circ q^{t} \circ A d_{\sigma-1}=-\left(T_{\sigma} R_{\sigma^{-1}}\right)^{t} \circ A d_{\sigma}^{*} \circ q \circ A d_{\sigma-1} \\
& =-\left(T_{\sigma} R_{\sigma^{-1}}\right)^{t} \circ q^{\sigma^{-1}}=-\left(T_{\sigma} R_{\sigma^{-1}}\right)^{t} \circ(q+\delta(Q(\sigma)))
\end{aligned}
$$

From equality $\Omega^{b}=q^{+}-q^{-}$and, from the fact that the morphism $\left(\tilde{r}^{+}\right)^{\sharp} \circ q^{+}\left(\operatorname{resp} . q^{-} \circ\left(\tilde{r}^{-}\right)^{\sharp}\right)$ is nothing but the identity map of $T_{\sigma} G$ (resp. $T_{\sigma}^{*} G$ ), at each point $\sigma$ of $G$, we get $\pi^{\sharp}=-\left(\tilde{r}^{+}\right)^{\sharp} \circ \Omega^{b} \circ\left(\tilde{r}^{-}\right)^{\sharp}$

Noticing the relation $Q(\sigma)=-A d_{\sigma}^{*} Q\left(\sigma^{-1}\right)$ first we have for each $x \in \mathcal{G}$

$$
\begin{gathered}
\delta(Q(\sigma))(x):=a d_{x}^{*} Q(\sigma)=-\left(a d_{x}^{*} \circ A d_{\sigma}^{*}\right) Q\left(\sigma^{-1}\right) \\
=-\left(A d_{\sigma}^{*} \circ a d_{\left(A d_{\sigma^{-1}} x\right)}^{*}\right) Q\left(\sigma^{-1}\right) .
\end{gathered}
$$

Second, we obtain

$$
\begin{aligned}
\left(A d_{\sigma}^{*} \circ \delta\left(Q\left(\sigma^{-1}\right)\right) \circ A d_{\sigma^{-1}}\right)(x) & =A d_{\sigma}^{*}\left(\delta\left(Q\left(\sigma^{-1}\right)\right)\left(A d_{\sigma^{-1}} x\right)\right) \\
& =A d_{\sigma}^{*} \circ a d_{\left(A d_{\left.\sigma^{-1} x\right)}^{*}\right.}^{*}\left(Q\left(\sigma^{-1}\right)\right) .
\end{aligned}
$$

This proves the following

$\delta(Q(\sigma))=-A d_{\sigma}^{*} \circ \delta\left(Q\left(\sigma^{-1}\right)\right) \circ A d_{\sigma^{-1}}$

Now, let's prove, using formulas above, that

$T_{\sigma} Q \circ \pi_{\sigma}^{\sharp} \circ\left(T_{\sigma} Q\right)^{t}$

only depends on $Q(\sigma)$. Formulas $(\mathrm{h})$, (i) and $(\mathrm{j})$ enable to write

$$
\begin{aligned}
T_{\sigma} Q \circ \pi_{\sigma}^{\sharp} \circ\left(T_{\sigma} Q\right)^{t}= & A d_{\sigma}^{*} \circ q \circ T_{\sigma} L_{\sigma-1} \circ\left(\tilde{r}^{+}\right)^{\sharp} \circ \Omega^{b} \circ\left(\tilde{r}^{-}\right)^{\sharp} \\
& \circ\left(T_{\sigma} R_{\sigma^{-1}}\right)^{t} \circ(q+\delta(Q(\sigma)))
\end{aligned}
$$

Apply now (b') and (f) to get

$$
\begin{aligned}
T_{\sigma} Q \circ \pi_{\sigma}^{\sharp} \circ\left(T_{\sigma} Q\right)^{t}= & -A d_{\sigma}^{*} \circ q \circ T_{\sigma} L_{\sigma-1} \circ\left(T_{\epsilon} L_{\sigma} \circ r \circ\left(T_{\epsilon} L_{\sigma}\right)^{t}\right) \\
& \circ^{t}\left(T_{\sigma} L_{\sigma-1}\right) \circ \delta\left(Q\left(\sigma^{-1}\right)\right) \circ T_{\sigma} L_{\sigma-1} \\
& \circ\left(T_{\epsilon} R_{\sigma} \circ r \circ\left(T_{\epsilon} R_{\sigma}\right)^{t}\right) \circ\left(T_{\sigma} R_{\sigma^{-1}}\right)^{t} \circ(q+\delta(Q(\sigma)))
\end{aligned}
$$

Simplifying this, one obtains

$$
T_{\sigma} Q \circ \pi_{\sigma}^{\sharp} \circ\left(T_{\sigma} Q\right)^{t}=-A d_{\sigma}^{*} \circ \delta\left(Q\left(\sigma^{-1}\right)\right) \circ A d_{\sigma-1} \circ r \circ(q+\delta(Q(\sigma)))
$$

Last, the equality $(\mathrm{k})$ allows us to conclude that the wanted expression (a) reads

$T_{\sigma} Q \circ \pi_{\sigma}^{\sharp} \circ\left(T_{\sigma} Q\right)^{t}=\delta(Q(\sigma)) \circ r \circ(q+\delta(Q(\sigma)))$

and then only depends on $Q(\sigma)$. This proves lemma 4.1.1.

\section{b. Proof of lemma 4.1 .2}

From lemma 4.1.1, the tensor $\pi$ can be projected by $Q$, into a Poisson tensor $\Lambda=Q_{*} \pi$ on $\operatorname{Im} Q$, whose associated vector bundles morphism is $\Lambda_{Q(\sigma)}^{\sharp}=T_{\sigma} Q \circ \pi_{\sigma}^{\sharp} \circ\left(T_{\sigma} Q\right)^{t}$ on the tangent fiber over any point $Q(\sigma)$ of $\operatorname{Im} Q$. Let $f$ and $g$ be in $\mathcal{C}^{\infty}(\operatorname{Im} Q)$, if $\{f, g\}_{\Lambda}$ is the Poisson bracket of $f$ and $g$; using expression (l) and the definition of $\delta$, we establish the following.

$$
\begin{aligned}
\{f, g\}_{\Lambda}(Q(\sigma))= & <(\Lambda)^{\sharp}(d f(Q(\sigma))), d g(Q(\sigma))> \\
= & <\delta(Q(\sigma))(r(q+\delta(Q(\sigma)))(d f(Q(\sigma))), d g(Q(\sigma))> \\
= & <\delta(Q(\sigma))(d f(Q(\sigma))), d g(Q(\sigma))> \\
& +<\delta(Q(\sigma))(r \circ \delta(Q(\sigma)))(d f(Q(\sigma))), d g(Q(\sigma))> \\
= & -<Q(\sigma),[x, y]>+<\delta(Q(\sigma))\left(r\left(a d_{x}^{*} Q(\sigma)\right), y>\right. \\
= & -<Q(\sigma),[x, y]>-<\delta(Q(\sigma))(y), r\left(a d_{x}^{*} Q(\sigma)\right)> \\
= & -<Q(\sigma),[x, y]>-r\left(a d_{x}^{*} Q(\sigma), a d_{y}^{*} Q(\sigma)\right. \\
= & -<Q(\sigma),[x, y]>-r\left(a d_{f}^{*} Q(\sigma), a d_{g}^{*} Q(\sigma)\right. \\
= & -<Q(\sigma),[x, y]>-<\left(a d_{x} \otimes a d_{y}\right) r, Q(\sigma) \otimes Q(\sigma)>.
\end{aligned}
$$


Here $d f(Q(\sigma))=: x$ and $d g(Q(\sigma))=: y$ are seen as elements of the Lie algebra $\mathcal{G}$.

If $f$ and $g$ are the restrictions to $\operatorname{Im} Q$ of some linear functions (also denoted $f$ and $g$ respectively) on $\mathcal{G}^{*}$, we have $d f(Q(\sigma)=f=: x, d g(Q(\sigma)=g=: y$ and the two last equalities above then read

$$
\begin{aligned}
\{f, g\}_{Q_{*}(\pi)}(Q(\sigma)) & =-<Q(\sigma),[f, g]>-r\left(a d_{f}^{*} Q(\sigma), a d_{g}^{*} Q(\sigma)\right. \\
& =-<Q(\sigma),[f, g]>-<\left(a d_{f} \otimes a d_{g}\right) r, Q(\sigma) \otimes Q(\sigma)>.
\end{aligned}
$$

This proves lemma 4.1.2.

To end this section, let's point out a consequence of the above theorem 4.0.5. We will use the same notations as in theorem 4.0.5. Let $F_{\sigma}$ be the symplectic leaf, through $\sigma \in G$, of the Poisson Lie group $\left(G, \pi:=r^{+}-r^{-}\right)$. The vector space $T_{\sigma} F_{\sigma}$ tangent to $F_{\sigma}$, at the point $\sigma$, is given by $T_{\sigma} F_{\sigma}=$ $T_{\epsilon} R_{\sigma}\left\{\eta(\sigma)\left(A d_{\sigma}^{*} \alpha,.\right), \alpha \in \mathcal{G}^{*}\right\}$. Here we have $\eta(\sigma):=A d_{\sigma} \tilde{r}-\tilde{r}$ and $R_{\sigma}$ is the right multiplication by $\sigma$ in $G$.

Notice the following relation $\eta(\sigma)\left(A d_{\sigma}^{*} \alpha,.\right)=A d_{\sigma}\left(r\left(A d_{\sigma}^{*} \alpha\right)\right)$. Due to formula (h), one has $T_{\sigma} Q\left(T_{\sigma} F_{\sigma}\right)=$ $\left\{a d_{\left(r \circ A d_{\sigma}^{*}\right) \alpha}^{*} Q(\sigma), \quad \alpha \in \mathcal{G}^{*}\right\}$.

As $r \circ A d_{\sigma}^{*}: \mathcal{G}^{*} \rightarrow \mathcal{G}$ is a vector spaces isomorphism, $T_{\sigma} Q$ bijectively maps $T_{\sigma} F_{\sigma}$ onto the orbit of $Q(\sigma)$ for the $\mathcal{G}$-coadjoint action.

Namely, we have

Corollary 4.2.1. Let $\left(G, \omega^{+}\right)$be a connected and simply connected symplectic Lie group. Put $q(x):=$ $i_{x} \omega, x \in \mathcal{G}:=\operatorname{Lie}(G)$ and $r=q^{-1}$, define $Q$ as above.

The leaves of (the symplectic foliation of) the Poisson Lie group $\left(G, \pi=r^{+}-r^{-}\right)$are coverings over the traces on ImQ of the coadjoint orbits of $G$. In particular, if $\omega^{+}$is exact, those symplectic leaves are coverings over coadjoint orbits of $G$.

There is a bijection between the Lie subgroup $G_{\pi}=\left\{\sigma \in G, \pi_{\sigma}=0\right\}$ of $G$, and the intersection of $\operatorname{Im} Q$ and the vector subspace $\left\{\alpha \in \mathcal{G}^{*}\right.$ s.t. $\alpha$ is invariant relative to the coadjoint representation $\}$ of $G$.

\section{References}

[1] Alekseevsky, D. V.; Perelomov, A. M. Poisson and symplectic structures on Lie algebras. I. J. Geom. Phys. 22 (1997), no. 3, 191-211.

[2] Alekseevsky, D.; Grabowski, J.; Marmo, G.; Michor, P. W. Poisson structures on double Lie groups. J. Geom. Phys. 26 (1998), no. 3-4, 340-379.

[3] Chi, Q.-S.; Merkulov, S. A.; Schwachhfer, L.J. On the existence of infinite series of exotic holonomies. Invent. Math. 126 (1996), no. 2, 391-411.

[4] Chu, Bon-Yao, Symplectic homogeneous spaces. Transactions of the AMS, 197 (1974), 145-159.

[5] Bordemann, M. Generalized Lax pairs, the modified classical Yang-Baxter equation, and affine geometry of Lie groups. Comm. Math. Phys. 135 (1990), no. 1, 201-216.

[6] Dardié, J.M. et Medina, A., Double extension symplectique d'un groupe de Lie symplectique. Adv. Math 117, (1996), 208-227.

[7] Dardié, J.M. et Medina, A., Algèbres de Lie kahlériennes et double extension. J. Algebra 185 (1996) 774-795.

[8] Diatta, A. et Medina, A., Espaces de Poisson homognes d'un groupe de Lie-Poisson. C. R. Acad. Sci. Paris Sér. I Math. 328 no 8 (1999), 687-690.

[9] Diatta, A., Poisson and Contact Geometry of Homogeneous Spaces. Ph.D. Thesis of Mathematics (in French). University Montpellier 2, France (2000).

[10] Dorfmeister, J., Nakajima, K., The fundamental conjecture for homogeneous Kähler manifolds. Acta Math 161, no. 1-2, 23-70, (1988). 
[11] Drinfeld, V.G., Hamiltonian structures on Lie groups, Lie bialgebras and the geometric meaning of the classical Yang-Baxter equations. Soviet Math. Dokl. 27, (1983), 68-71.

[12] Fried, D.; Goldman, W.; Hirsch, M.W., Affine manifolds and solvable groups. Bull. Amer. Math. Soc. (N.S.) 3 (1980), no. 3, 1045-1047.

[13] Helmstetter, J., Radical d'une algèbre à gauche. Ann. Inst. Fourier 29, no 4, (1979), 17-35.

[14] Koszul, J.L., Sur la forme hermitienne canonique des espaces homogènes complexes. Canad. J. Math 7 (1955), 562-576.

[15] Koszul, J. L., Domaines bornés homogènes et orbites de groupes de transformations affines. Bull. Soc. Math. France 89, (1961), 515-533.

[16] Lichnerowicz, A., Les variétés de Poisson et leurs algèbres de Lie associes. J. Dif. Geom. 12 (1977), no. 2, 253-300.

[17] Lichnerowicz, A. et Medina, A., On Lie groups with left invariant symplectic or kahlerian structures. Letters Math. Phy. 16, 225-335, (1988).

[18] Lichnerowicz, A. et Medina, A., Groupes à structures symplectiques ou khleriennes invariantes. C. R. Acad. Sci. Paris Sr. I Math. 306 (1988) no. 3, 133-138.

[19] Lichnerowicz, A., Les groupes Kahlériens, in Symplectic Geometry and Mathematical Physics (P. Donato et al., Eds.), 245-259, progr. Math. 99. Birkhauser, Boston/Basel/Berlin, (1991).

[20] Lu, J.-H. and Weinstein, A., Poisson Lie groups, dressing transformations, and Bruhat decompositions. J. Diff. Geom. 31, (1990) 501-526.

[21] Medina Perea, A., Flat left-invariant connections adapted to the automorphism structure of a Lie group. J. Differential Geom. 16 (1981), no. 3, 445-474, (1982).

[22] Medina, A. et Revoy, Ph., Groupes de Lie à structure symplectique invariante. Symplectic geometry, groupoids and integrable systems, in "Séminaire Sud Rodhanien", M.S.R.I, 247-266, Springer-Verlag, New York/Berlin (1991).

[23] Milnor, J. On fundamental groups of complete affinely flat manifolds. Advances in Math. 25 (1977), no. $2,178-187$.

[24] Nijenhuis, A., Sur une classe de propriétés communes à quelques types différents d'algèbres. Enseignement Math. (2) 14, 1968, 225-277, (1970).

[25] Semenov Tian Shansky, M. A., What a classical r-matrix is. Funct. Anal. Appl. 17 (1983) no.4, 17-33.

[26] Vinberg E. B., Convex homogeneous cones. Transl. Moscow Math. Soc. 12, (1963), 340-403.

[27] Weinstein, A., The local structure of Poisson manifolds, J. Diff. Geom. 18, (1983), 523-557.

Departement of mathematics. National University of Ireland, Maynooth. Co. Kildare, Ireland.

UMR 5030 du CNRS. Département de Mathématiques.

Université Montpellier 2, 34095 Montpellier cedex 5.

e.mail adresses: adiatta@maths.may.ie; or andrediatta@hotmail.com;

medina@darboux.math.univ-montp2.fr 